nóesis Determinantes de la ocupación hotelera de los principales... / Volumen 29, Número 58-1 agosto - diciembre 2020 www.revistanoesis.mx 84

\title{
DETERMINANTES DE LA OCUPACIÓN HOTELERA DE LOS PRINCIPALES DESTINOS DE SOL Y PLAYA DE MÉXICO
}

\author{
Determinants of hotel occupancy of the main destinations \\ of sun and beach in Mexico
}

Reyna María Ibañez Pérez ${ }^{1}$ Marco Antonio Almendarez-Hernández², Ismael Sánchez-Brito ${ }^{3}$

Fecha de recepción: 3 de septiembre de 2019.

Fecha de aceptación: 9 de enero de 2020.

1- Nacionalidad: Mexicana. Grado: Doctorado en Economía. Adscripción: Universidad Autónoma de Baja California Sur.. Correo electrónico: ribanez@uabcs.mx. (D) ORCID: http://orcid.org/0000-0002-9392-3490

2- Nacionalidad: Mexicana. Grado: Doctorado en Economía. Adscripción: Universidad Autónoma de Baja California Sur. Correo electrónico: malmendarez@cibnor.mx. (iD ORCID: http://orcid.org/0000-0002-6017-3929

3- Nacionalidad: Mexicana. Grado: Doctorado en Ciencias Marinas y Costeras. Adscripción: Universidad Autónoma de Baja California Sur. Correo electrónico: isanchez@cibnor.mx. (D) ORCID: http://orcid.org/0000-0003-3811-0814 


\section{Resumen}

En México, el crecimiento de la ocupación hotelera es asimétrico. En este trabajo, se analiza el comportamiento de diferentes variables turísticas a fin de identificar los determinantes de la ocupación hotelera en los principales sitios de sol y playa. Con datos del panel desbalanceado y el estimador FGLS se examina, empíricamente, la función de la tasa de ocupación. Con ello, se corrobora que en los lugares con mayor afluencia y donde los viajeros prolongan más su permanencia, son más proclives a mostrar mejor desempeño en la variable analizada. Aunque se sabe que los establecimientos de tres y cuatro estrellas contribuyen en mayor medida, las estrategias de crecimiento de la infraestructura hotelera con estas categorías son relevantes debido al aumento en la demanda que han experimentado en el periodo estudiado. Sin embargo, es necesario que no se desatiendan políticas encaminadas a la atracción de consumidores a los hoteles de cinco y dos estrellas.

Palabras clave: Ocupación, Destinos de playa, Llegadas a hoteles, Estadía, Datos de panel.

\section{Abstract}

In México, the growth of hotel occupancy is asymmetric. In this work, the behavior of different tourist variables is analyzed in order to identify the determinants of hotel occupancy in the main sun and beach sites. With unbalanced panel data and the FGLS estimator, the function of the occupancy rate was examined empirically. It was corroborated that that in places with greater affluence and where travelers prolong their permanence, they are more inclined to show better performance in the variable analyzed. However, three- and four-star establishments contribute to a greater extent. In this sense, the strategies of growth of the hotel infrastructure of three and four stars are relevant due to the increase in demand that they have experienced in the period studied, as long as they do not neglect policies that consist of attracting consumers to hotels of five and two stars.

Keywords: Occupation, Beach destinations, Hotel arrivals, Stay, Panel data. 


\section{Introducción}

$\mathrm{M}$ éxico cuenta con 11,122 kilómetros de costas (Secretaría del Medio Ambiente y Recursos Naturales-SEMARNAT, 2010). Sus extensos litorales proveen atractivos para la realización de actividades como el turismo, sector que en la actualidad constituye la tercera fuente de divisas (Secretaría de Hacienda y Crédito Público-SHCP y Banco Nacional de Comercio ExteriorBANCOMEXT, 2016).

Esto, sumado a la introducción de otras medidas para fortalecer la economía turística, ha intensificado la promoción de los "centros de playa", que, según la Secretaría de Turismo (SECTUR, 2014), son un medio para satisfacer las exigencias de prácticas asociadas al segmento de sol y playa, puntal en la generación de recursos para el país.

Una de las acciones gubernamentales más relevantes en este sentido fue la creación de polos de desarrollo turístico a finales de los años sesenta. Denominados "Centros Integralmente Planeados" (CIP), su propósito fue activar la economía en zonas del territorio nacional donde existían pocas opciones de producción e ingreso (Tulio y Santamaría, 2015).

En un principio se establecieron cuatro CIP: Cancún, Los Cabos, Loreto e Ixtapa; sin embargo, 10 años después, se agregó a la lista Huatulco (Benseny, 2007; Bringas, 1999; Coll-Hurtado, 2016).

Según apunta Bringas (1999), más adelante, con la creación del Fondo Nacional de Fomento al Turismo (FONATUR), también se brindó apoyo a aquellos destinos de playa con mayor consolidación, experiencia y grado de madurez en el rubro.

Más recientemente se han generado estrategias para aprovechar el potencial turístico de las zonas costeras del país. A ello se suman iniciativas como el Programa de Centros de Playa, que atiende directamente a estados con litorales que poseen un alto potencial turístico, con objeto de contribuir a su desarrollo equilibrado.

No obstante, si bien son una fuente importante de divisas y generación de empleos porque mantienen tasas de ocupación más altas que otros sitios turísticos (Datatur, 2019); existen evidencias sobre sus múltiples problemáticas a nivel local y las disparidades que presentan con otros destinos de su misma categoría 4 .

Por ejemplo, en el trabajo de Amaya et al. (2017) se analizan ciertos agentes clave de variables que influyen en la competitividad. Éste se centra en las diferencias que presentan dos destinos masivos: Manzanillo y Cancún. El estudio, de carácter comparativo, concluye que el primero resulta menos competitivo y presenta un nivel de bienestar más bajo, con relación al segundo.

Según datos oficiales, Cancún presenta una actividad turística más intensa, reflejo de su evolución en indicadores como: número de cuartos, hoteles y moteles; la estadía promedio y el porcentaje de ocupación.

Aunque pueda resultar inviable hacer comparaciones entre dos destinos turísticos tan asimétricos, la investigación de Amaya et al. (2017) es parte importante del estado del arte y merece ser tomada como una referencia.

Del mismo modo, Ibáñez (2017), quien no sólo revisa indicadores de competitividad ya establecidos, sino que propone otros nuevos, corrobora que las zonas turísticas donde existe mayor ingreso, ocupación hotelera y permanencia de visitantes no siempre registran un mayor nivel de bienestar.

4- Datatur es un sistema alojado en bases de datos de la SECTUR, donde se recaba, procesa y difunde información básica del turismo en México. Dentro de sus estadísticas, se divide a los centros de playa en CIP y tradicionales (Datatur, 2019). 
Además, dentro del mismo trabajo aparece un estudio de variables básicas en destinos de la región Noroeste, donde se señala que los impactos del turismo en costas mexicanas son diversos y la evolución de este sector se relaciona con aspectos de distinta naturaleza. Esto quiere decir que cada paraje turístico presenta características únicas y particulares (Ibáñez, 2016; 2017).

Lo anterior es de suma importancia para los tomadores de decisiones, pues es necesario que atiendan estos factores si desean desarrollar una planeación turística eficiente, responsable y con visión a largo plazo.

Por su parte, Dávila (2015) argumenta que los primeros CIP revelan diferentes grados de madurez; incluso, algunos presentan un crecimiento exponencial. Pero aun con los esfuerzos y acciones implementadas, destinos como Loreto siguen sin alcanzar las expectativas iniciales.

De este modo, se observa un crecimiento asimétrico de los centros de playa, asociado al comportamiento diferenciado de indicadores turísticos representativos. Tal como se aprecia en la gráfica 1, existe una notoria concentración de la oferta de hospedaje en destinos como Puerto Vallarta, Acapulco, Cancún y Mazatlán.

Gráfica 1. No. de establecimientos de hospedaje registrados en destinos de playa de México seleccionados, 2016

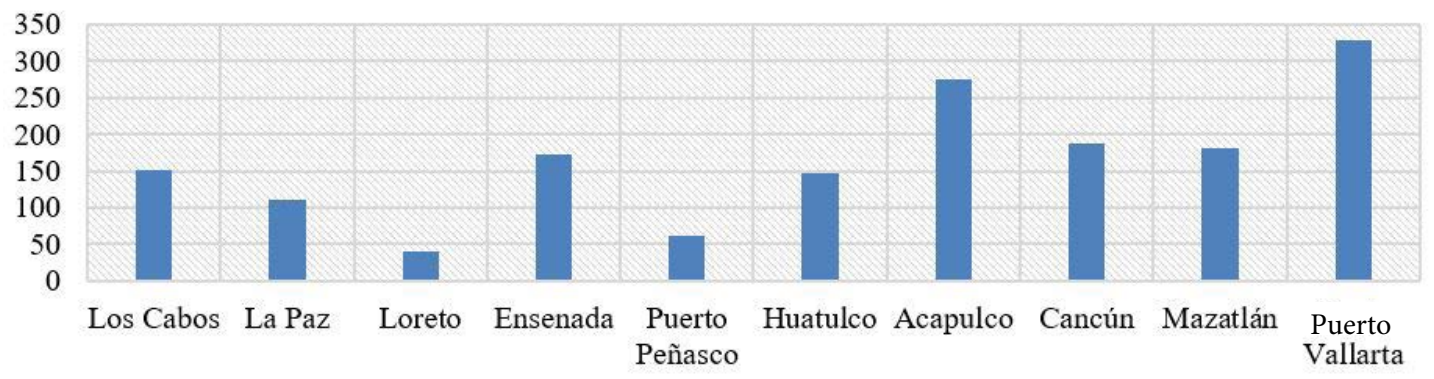

Fuente: Elaboración propia con base en datos del INEGI y Datatur.

Además, en destinos de sol y playa predominan unidades de hospedaje de cinco y cuatro estrellas, como se registra a continuación. 
Gráfica 2.

Cuartos y unidades de hospedaje registrados en destinos de playa seleccionados de México, según categoría, 2016

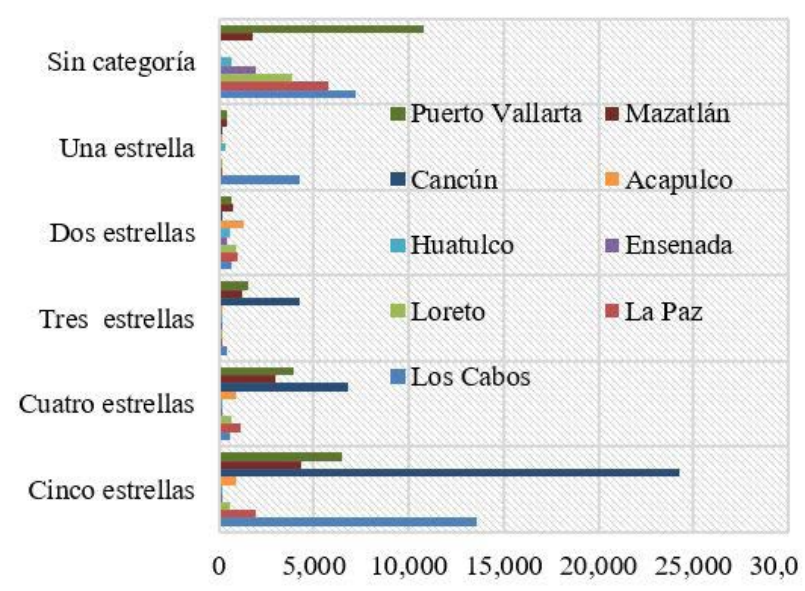

\section{Gráfica 3.}

Distribución de cuartos y unidades de hospedaje registrados en destinos de playa seleccionados de México, según categoría, 2016

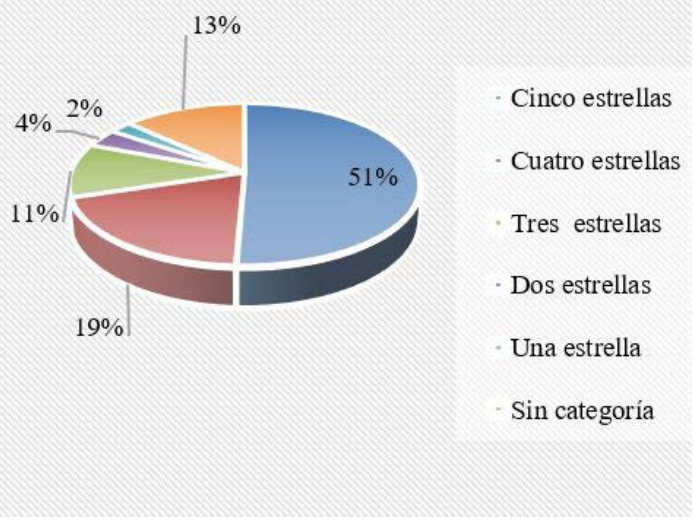

Fuente: Elaboración propia con base en datos del INEGI y Datatur.

Asimismo, a partir de datos detallados para diez destinos de playa, se aprecia que en $2016^{5}$ (último año de la serie de tiempo analizada), más de $70 \%$ de la afluencia de visitantes se concentra en tres sitios: Cancún, Acapulco y Puerto Peñasco. Lo anterior se expone en la siguiente gráfica.

Gráfica 4. Afluencia de visitantes en destinos de playa de México seleccionados, 2016

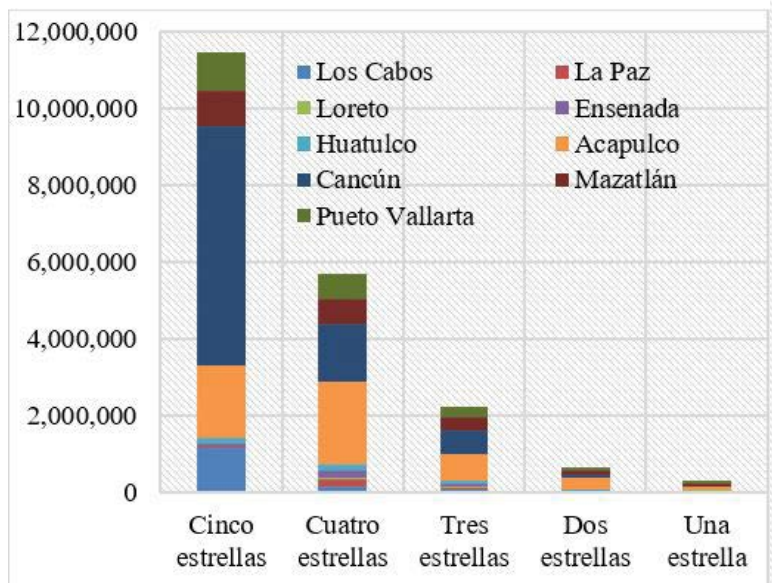

Gráfica 5. Distribución de la afluencia total de visitantes en destinos de playa de México seleccionados, 2016

Fuente: Elaboración propia con base en datos del INEGI y Datatur. 
En tanto, en la gráfica 5 se observa que el mayor porcentaje de personas tiene predilección por establecimientos de cinco y cuatro estrellas.

Con relación a la estadía promedio, se puede corroborar en el cuadro 1 que ésta oscila entre 1.30 noches por visitante en Acapulco y 4.7 en el caso de Los Cabos.

Cuadro 1. Estadía promedio en destinos de playa de México seleccionados, 2016

\begin{tabular}{|c|c|c|c|c|c|c|c|c|}
\hline $\begin{array}{c}\text { Los } \\
\text { Cabos }\end{array}$ & La Paz & Loreto & Ensenada & Huatulco & Acapulco & Cancún & Mazatlán & $\begin{array}{c}\text { Puerto } \\
\text { Vallarta }\end{array}$ \\
\hline 4.7 & 2.1 & 1.90 & 1.40 & 3.90 & 1.30 & 3.20 & 2.80 & 3.50 \\
\hline
\end{tabular}

Fuente: Elaboración propia con base en datos de INEGI.

Pese a la asimetría que existe entre indicadores representativos en destinos de sol y playa, no se tienen registros sobre investigaciones que, a partir de información y un análisis econométrico, identifiquen la relación que guardan variables clave como la llegada de turistas por categoría de hotel y arribo de turistas por cuarto y por tipo de estrato de establecimiento con la tasa de ocupación hotelera.

En función de esta problemática y la relevancia para el desarrollo turístico, se contempla analizar el comportamiento de variables turísticas para identificar los determinantes de la ocupación hotelera en los principales destinos de sol y playa, que son Los Cabos, La Paz, Loreto, Ensenada, Puerto Peñasco, Huatulco, Acapulco, Cancún, Mazatlán y Puerto Vallarta.

Asimismo, a partir de la revisión de referencias especializadas, se pretende responder al siguiente planteamiento: ¿cuál es la magnitud de la respuesta de la tasa de ocupación hotelera ante los cambios en la llegada de turistas por categoría de hotel, arribo de turistas por cuarto de hotel, estadía y ciertas variables de control como el Índice de Precios al Consumidor (IPC) y Producto Interno Bruto (PIB), en los principales destinos de sol y playa de México?

A manera de hipótesis, se plantea que esta clase de destinos en México (a los que acude un mayor número de turistas a hoteles por categoría) registran más viajeros por cuarto, según estrato de establecimiento; alargan más su estadía en los centros turísticos y aportan en mayor magnitud a la tasa de ocupación.

A fin de cumplir con los objetivos, se emplea información estadística que se encuentra estructurada en datos de panel desbalanceado, dentro de un periodo que va de 2000 a 2016.

El método econométrico que se utiliza para analizar empíricamente la función de la tasa de ocupación es el de Mínimos Cuadrados Generalizados Factibles (FGLS, por sus siglas en inglés). Todas las variables independientes fueron transformadas en logaritmos naturales y son interpretadas como elasticidades.

Finalmente, la información al interior del artículo se encuentra organizada en seis apartados. El primero corresponde a esta introducción, donde se exponen aspectos medulares de la investigación, como su relevancia, la problemática y sus objetivos. En el apartado dos se revisa la literatura referente a estudios que abordan la estadía promedio y la oferta del número de cuartos disponibles. En el tercero, se detallan las técnicas y procedimientos cuantitativos empleados para corroborar la hipótesis y responder a la pregunta de investigación. En el cuarto, las consideraciones más relevantes, así como su discusión. En el quinto apartado se destacan las principales conclusiones y, en el último, se enlista la bibliografía consultada. 


\section{Revisión de literatura}

Actualmente, en México no se tiene referencia de alguna investigación que, con base en técnicas econométricas, estudie los diferentes factores que afectan la tasa de ocupación. Parte de los estudios a nivel nacional son más bien de carácter general.

Por ejemplo, está el de Guzmán-Soria et al. (2011), que analiza los determinantes de la demanda internacional del turismo "de forma global". También aparecen trabajos orientados a un solo sitio, como el de Cruz-Chávez et al. (2016), donde se examina específicamente al turismo extranjero en el CIP Los Cabos.

Sin embargo, a nivel internacional sí se pueden encontrar trabajos de amplio interés en el tema (cuadro 2). En primera instancia, aparece el de Jeffrey y Barden (2000), que utiliza el método de análisis de serie de tiempo de la ocupación hotelera en el Reino Unido para monitorear la actividad hotelera. Con el procedimiento estadístico de análisis factorial, considera cuatro variables para su estudio: nivel de ocupación, estacionalidad, tendencia a largo plazo y duración de la temporada. Mediante esta dinámica, trata de puntualizar diferentes políticas de segmentación de la oferta hotelera.

Además, los autores cuentan con otro trabajo (Jeffrey y Barden, 2001) que utiliza cuatro modelos multivariados en la tendencia de ocupación hotelera para dicha nación: la ocupación general, estacionalidad, tendencia a largo plazo y duración de la temporada; así como otras características de los patrones de ocupación.

También se encuentra la investigación de Qu et al. (2002), donde se utiliza un modelo de ecuaciones simultáneas para definir la oferta y demanda de cuartos de hotel en Hong Kong. Dicho proyecto concluye que las variables del precio de cuarto de hotel y el arribo de turistas son los factores que determinan la demanda de cuartos.

Por su parte, Jang y Lu (2002) hacen un ejercicio comparativo de un análisis del retorno de inversión de dos hoteles: uno comercial y otro que incluye casino en los Estados Unidos de América (EUA). Los resultados sugieren que el tamaño del establecimiento no es un factor causal que afecte el retorno de la inversión, pero sí la deuda de los hoteles comerciales.

En otro estudio hecho por Mutlag y Mzaiel (2003) para examinar los componentes de la tasa de ocupación en los hoteles de Amman, Jordania, se consideran las características del establecimiento como factor; es decir, la localización, tamaño y número de cuartos.

También se registra una investigación que usa el análisis estacional de las tasas de ocupación en Gales, donde se utiliza la técnica de análisis de componentes principales para segmentar la oferta hotelera. Los resultados muestran que los hoteles con más estrellas tuvieron éxito para alargar su temporada principal en primavera y, especialmente, en otoño (Koenig y Bischoff, 2004).

A partir de la liberalización de los juegos de azar en Macao, China, Lam y Lei (2010) establecen un modelo que toma en cuenta factores microeconómicos y macroeconómicos para determinar la tasa de ocupación promedio por tipo de categoría. Se encontró que existe una relación negativa entre los casinos y la tasa de ocupación.

En tanto, en una investigación de Ging (2010) para estimar los factores de las tarifas de cuartos de hotel en Singapur, aparece que el arribo de turistas es positivo; aunque los efectos de actos terroristas en países vecinos repercuten de forma negativa. 
De igual forma está el trabajo de O’Neill (2011), que examina la estabilización de la oferta hotelera en EUA a partir del tipo de hotel, localización, tamaño y nivel de servicio.

El mismo Ging (2011) estima los determinantes de la demanda para la tasa de ocupación hotelera en Hong Kong. Según el estudio, son los ingresos de los turistas los que intervienen en la oferta hotelera (dependiendo de su categoría), así como la influencia en las fluctuaciones del tipo de cambio.

Dentro de la literatura también se encuentra un modelo Rasch aplicado para el turismo internacional en Taiwán, que incluye cinco variables: la tasa de ocupación promedio, tarifa promedio del cuarto, valor de la producción promedio por empleado y por cuarto o departamento, la producción promedio por empleado por categoría de departamento y la relación de ingresos y gastos. La finalidad es obtener un mejor desempeño y aumento del rendimiento de las empresas hoteleras (Peng et al., 2013).

Asimismo, existe un modelo de tasa de ocupación hotelera en Macao, donde se exhibe una correlación positiva entre la tasa de ocupación y dos variables independientes: el total de cuartos disponibles y el número de turistas que arriban (Lei y Lam, 2015).

A partir de un enfoque cualitativo, una investigación en la isla de Santa Lucía, ubicada en el mar Caribe, determina que el aumento de la tasa de ocupación depende del modelo operativo del hotel y los servicios que ofrece para cumplir con las expectativas del cliente (Sean, 2016).

A su vez, Mei Fung et al. (2016) estiman la función de demanda en Hong Kong con fluctuaciones anticipadas en ocupación hotelera. Una de las variables fue el ingreso de los turistas que, de acuerdo al estudio, impacta de diferentes maneras en función de la categoría del hotel.

En tanto, en EUA se hizo un pronóstico de ocupación hotelera de diez mercados geográficos, con dos técnicas de series de tiempo: Box-Jenkins y el aditivo Holt-Winters, con el fin de prever la demanda futura de la ocupación (Warren, 2017).

Por último, está Arbelo et al. (2018), quienes, a partir de un modelo de frontera estocástico bayesiano, determinan la eficiencia y rentabilidad hotelera en España, con variables como tamaño del hotel, ubicación, tasa de ocupación por región, satisfacción del cliente, entre otras.

Cuadro 2. Estudios realizados que determinan la tasa de ocupación hotelera

\begin{tabular}{|c|c|c|c|c|c|}
\hline Autor & Año & País & Variables & Objetivo & Modelo \\
\hline $\begin{array}{c}\text { Jeffrey y Bar- } \\
\text { den }\end{array}$ & 2000 & $\begin{array}{c}\text { Reino } \\
\text { Unido }\end{array}$ & $\begin{array}{c}\text { Nivel de ocupación, } \\
\text { estacionalidad, } \\
\text { tendencia de largo } \\
\text { plazo y duración de la } \\
\text { temporada }\end{array}$ & $\begin{array}{c}\text { Monitoreo de la } \\
\text { actividad hotelera }\end{array}$ & $\begin{array}{c}\text { Matriz de correlación y Análi- } \\
\text { sis de Varianza (ANOVA) }\end{array}$ \\
\hline $\begin{array}{c}\text { Jeffrey y Bar- } \\
\text { den }\end{array}$ & 2001 & $\begin{array}{c}\text { Reino } \\
\text { Unido }\end{array}$ & $\begin{array}{c}\text { Ocupación gene- } \\
\text { ral, estacionalidad, } \\
\text { tendencia a largo } \\
\text { plazo, duración de la } \\
\text { temporada }\end{array}$ & $\begin{array}{c}\text { Determinación } \\
\text { de los factores } \\
\text { de la ocupación } \\
\text { hotelera }\end{array}$ & Serie de tiempo, multivariado \\
\hline Jung y Yan & 2002 & $\begin{array}{c}\text { Hong } \\
\text { Kong }\end{array}$ & $\begin{array}{c}\text { Precio del cuarto, } \\
\text { arribo de turistas }\end{array}$ & $\begin{array}{c}\text { Equilibrio de } \\
\text { mercado }\end{array}$ & Ecuaciones simultáneas \\
\hline Estados & $\begin{array}{c}\text { Tamaño y categoría } \\
\text { del hotel, servicio de } \\
\text { juegos }\end{array}$ & $\begin{array}{c}\text { Retorno de inver- } \\
\text { sión }\end{array}$ & Test t y ANOVA \\
\hline
\end{tabular}




\begin{tabular}{|c|c|c|c|c|c|}
\hline $\begin{array}{c}\text { Koenig y Bis- } \\
\text { choff }\end{array}$ & 2004 & Gales & Categoría del hotel & $\begin{array}{c}\text { Segmentación de } \\
\text { mercado }\end{array}$ & $\begin{array}{l}\text { Componentes principales y } \\
\text { análisis de clúster }\end{array}$ \\
\hline $\begin{array}{l}\text { Mutlag y } \\
\text { Mzaiel }\end{array}$ & 2003 & Jordania & $\begin{array}{l}\text { Localización, tamaño } \\
\text { y número de cuartos }\end{array}$ & $\begin{array}{c}\text { Factores de la } \\
\text { tasa de ocupación }\end{array}$ & $\begin{array}{l}\text { Mínimos cuadrados ordina- } \\
\text { rios }\end{array}$ \\
\hline Lam y Lei & 2010 & Macao & $\begin{array}{l}\text { Factores micro y ma- } \\
\text { croeconómicos }\end{array}$ & $\begin{array}{l}\text { Segmentación de } \\
\text { la oferta hotelera }\end{array}$ & ANOVA, Regresión lineal \\
\hline Ging & 2010 & Singapur & $\begin{array}{l}\text { Arribo de turistas, } \\
\text { actividades terroristas }\end{array}$ & $\begin{array}{l}\text { Determinación } \\
\text { de las tarifas de } \\
\text { cuarto de hotel y } \\
\text { arribo de turistas } \\
\text { internacionales }\end{array}$ & $\begin{array}{l}\text { Mínimos cuadrados ordina- } \\
\text { rios }\end{array}$ \\
\hline Kim & 2010 & $\begin{array}{l}\text { Corea del } \\
\text { Sur }\end{array}$ & $\begin{array}{l}\text { Categoría de hotel, } \\
\text { casino, precio }\end{array}$ & $\begin{array}{l}\text { Determinación de } \\
\text { factores de la tasa } \\
\text { de ocupación }\end{array}$ & Precios hedónicos \\
\hline O’Neill & 2011 & $\begin{array}{l}\text { Estados } \\
\text { Unidos de } \\
\text { América }\end{array}$ & $\begin{array}{c}\text { Tipo de hotel, locali- } \\
\text { zación, tamaño y nivel } \\
\text { de servicio }\end{array}$ & $\begin{array}{l}\text { Estabilización de } \\
\text { la oferta hotelera }\end{array}$ & ANOVA \\
\hline Ging & 2011 & Singapur & $\begin{array}{l}\text { Ingresos de los } \\
\text { turistas, categoría del } \\
\text { hotel, tasa de cambio, } \\
\text { actividades terroristas }\end{array}$ & $\begin{array}{l}\text { Factores de las } \\
\text { tarifas de cuarto } \\
\text { de hotel }\end{array}$ & $\begin{array}{c}\text { Test Dickey-Fuller y Breusch- } \\
\text { Godfrey multiplicador } \\
\text { lagrangiano }\end{array}$ \\
\hline $\begin{array}{l}\text { Peng, Huang } \\
\text { y Wu }\end{array}$ & 2013 & Taiwán & $\begin{array}{c}\text { Tasa de ocupación } \\
\text { promedio, tarifa } \\
\text { promedio del cuarto, } \\
\text { valor de la producción } \\
\text { promedio por emplea- } \\
\text { do y por cuarto y la } \\
\text { relación de ingresos y } \\
\text { gastos }\end{array}$ & $\begin{array}{l}\text { Modelo de ocupa- } \\
\text { ción del turismo } \\
\text { internacional }\end{array}$ & Análisis envolvente de datos \\
\hline Lei y Lam & 2015 & Macao & $\begin{array}{l}\text { Número de cuartos } \\
\text { disponibles y número } \\
\text { de turistas que arriban }\end{array}$ & $\begin{array}{c}\text { Modelo de tasa } \\
\text { de ocupación } \\
\text { hotelera }\end{array}$ & Tests $t$ y ANOVA \\
\hline Mathew & 2016 & $\begin{array}{l}\text { Santa } \\
\text { Lucía }\end{array}$ & $\begin{array}{l}\text { Modelo operativo del } \\
\text { hotel y servicios que } \\
\text { ofrecen }\end{array}$ & $\begin{array}{l}\text { Nivel de satisfac- } \\
\text { ción del cliente }\end{array}$ & $\begin{array}{l}\text { Análisis cualitativo, triangu- } \\
\text { lación metodológica }\end{array}$ \\
\hline Mei Fung et al. & 2016 & $\begin{array}{l}\text { Hong } \\
\text { Kong }\end{array}$ & $\begin{array}{l}\text { Ingresos de los turis- } \\
\text { tas, categoría de los } \\
\text { hoteles }\end{array}$ & $\begin{array}{l}\text { Estimación de } \\
\text { la función de } \\
\text { demanda }\end{array}$ & Regresión logística \\
\hline Warren & 2017 & $\begin{array}{l}\text { Estados } \\
\text { Unidos de } \\
\text { América }\end{array}$ & $\begin{array}{l}\text { Tasa de ocupación } \\
\text { hotelera }\end{array}$ & $\begin{array}{c}\text { Pronóstico de la } \\
\text { actividad hotelera }\end{array}$ & Holt-Winters y Box-Jenkins \\
\hline $\begin{array}{l}\text { Arbelo, Arbelo- } \\
\text { Pérez y Pérez } \\
\text { Gómez }\end{array}$ & 2018 & España & $\begin{array}{l}\text { Tamaño de hotel, } \\
\text { ubicación, tasa de } \\
\text { ocupación por región, } \\
\text { satisfacción del cliente }\end{array}$ & $\begin{array}{c}\text { Determinación } \\
\text { de la eficiencia } \\
\text { y rentabilidad } \\
\text { hotelera } \\
\end{array}$ & $\begin{array}{l}\text { Frontera estocástico baye- } \\
\text { siano }\end{array}$ \\
\hline
\end{tabular}

Fuente: Elaboración propia. 
Aunque no es el eje particular de este trabajo, es relevante señalar que una de las grandes críticas a los destinos convencionales de sol y playa es su tendencia natural a la insostenibilidad (Gómez, 2011; Jiménez, 1988; Velarde, Santillán y Obombo, 2016). En parte, por el aumento desmesurado de la oferta, que pone en riesgo su propio desarrollo, al traer consecuencias que muchas veces son subestimadas y, desafortunadamente, en algunos casos también irreversibles.

Con relación a ello, Damián y Navarro (2005) revisan el impacto del turismo desde un ámbito poco abordado. En dicha evaluación citan como ejemplo los cambios socioculturales, el estrés emocional para el visitante y/o los residentes y la disminución de calidad de la experiencia turística. Señalan que estos factores, en conjunto con la masificación (propiciada por el aumento de la oferta), inciden potencialmente en la reducción de la afluencia e ingresos turísticos.

En cuanto a los impactos socioambientales del desarrollo turístico, Ibáñez (2017) manifiesta que, desde hace décadas, diversos actores discuten sobre el tema. Ello ha contribuido a la generación de nuevos indicadores y modelos que buscan evaluar el grado de sustentabilidad en regiones con o sin actividad turística, como la capacidad de carga.

No obstante, en la actualidad no existen umbrales plenamente consensuados sobre el número máximo de turistas, el total de hectáreas máximas de aprovechamiento turístico o número de unidades de hospedaje máximo que deben prevalecer en un sitio turístico; de tal suerte que se garantice un desarrollo benéfico para la comunidad receptora y los propios visitantes. Esta situación revela un fuerte reto en materia de planeación turística, con miras a alcanzar una sustentabilidad de largo plazo (Ibañez, 2017; Lime,1977; Lucas,1964; Madrid, 2015).

\section{Metodología}

\subsection{Planteamiento del modelo: la función de los determinantes de la ocupación hotelera}

Para evaluar econométricamente los efectos de la ocupación hotelera, la presente investigación se fundamenta en los modelos propuestos por Ching-chi y Weng (2010), Lei y Lam (2015), O'Neill (2011), Phillips et al. (2017) y Succurro y Boffa (2010). No obstante, se aplica una ligera modificación y adaptación en la metodología: en lugar de introducir el número de llegadas de turistas en forma agregada, se incorpora como variable el arribo de turistas a hoteles. Esta última se categoriza por establecimiento y toma en cuenta aquéllos que presentan una clasificación de dos hasta cinco estrellas.

A su vez, cada una de las variables se divide a partir de sus respectivas categorías de cuartos de hotel, con lo que se obtiene el número de llegadas por habitación para cada clasificación de establecimiento. Esto permite que el modelo sea más consistente y equilibrado.

De igual forma, se incluye la estadía promedio de los turistas en el sitio para explorar su influencia en la tasa de ocupación. Sobre esta cuestión, cabe precisar que aquellos destinos de sol y playa donde los turistas prolongan su estancia son más proclives a incrementar su tasa de ocupación.

Dada la naturaleza de la estructura de la base de datos, el modelo se expresa con la especificación semilogarítmica en datos de panel desbalanceado durante el periodo 2000-2016, de la siguiente forma: 
- Revista de Ciencias Sociales y Humanidades. ISSN-P: 0188-9834 ISSN-E:2395-8669.

$$
\begin{gathered}
O R_{i t}=\alpha_{1 i}+\beta_{1} \ln A T H 2_{i t}+\beta_{2} \ln A T H 3_{i t}+\beta_{3} \ln A T H 4_{i t}+\beta_{4} \ln A T H 5_{i t}+\beta_{5} \operatorname{lnS} T_{i t}+ \\
\beta_{6} \operatorname{lnIPC} C_{i t}+\beta_{7} \ln P I B_{i t}+e_{i t}
\end{gathered}
$$

Como la variable de ocupación en el destino de sol y playa (OR) se encuentra expresada en tasa, no fue convertida en logaritmos naturales; de lo contrario, ocasionaría problemas como la subestimación en los coeficientes de la regresión.

En la ecuación (1), los diferenciales en la tasa de ocupación se deben a las distintas llegadas de turistas por unidad de hotel, correspondientes a cada categoría. Quiere decir que todos los agentes se hospedan en el mismo número de cuartos. Una forma deseable sería controlar las llegadas de turistas por número de cuartos, función que se expresa a continuación:

$$
\begin{gathered}
O R_{i t}=\alpha_{1 i}+\beta_{1} \ln A T R 2_{i t}+\beta_{2} \ln A T R 3_{i t}+\beta_{3} \ln A T R 4_{i t}+\beta_{4} \ln A T R 5_{i t}+\beta_{5} \ln S T_{i t}+ \\
\beta_{6} \operatorname{lnIPC} C_{i t}+\beta_{7} \ln P I B_{i t}+e_{i t}
\end{gathered}
$$

\begin{tabular}{|c|c|c|c|}
\hline Variable & Descripción & $\begin{array}{l}\text { Efecto esperado } \\
\text { sobre la tasa de } \\
\text { ocupación }\end{array}$ & Fuente \\
\hline OR & Tasa de ocupación en el destino de sol y playa i en el tiempo $t$ & & $\begin{array}{c}\text { Secretaría de Turis- } \\
\text { mo, Datatur }\end{array}$ \\
\hline InATH2 & $\begin{array}{l}\text { Logaritmo natural del número de llegadas de turistas a } \\
\text { establecimientos de dos estrellas en el destino de sol y playa } \\
\text { i en el tiempo t }\end{array}$ & + & $\begin{array}{l}\text { Secretaría de Turis- } \\
\text { mo, Datatur }\end{array}$ \\
\hline InATH3 & $\begin{array}{l}\text { Logaritmo natural del número de llegadas de turistas a } \\
\text { establecimientos de tres estrellas en el destino de sol y playa } \\
\text { i en el tiempo t }\end{array}$ & + & $\begin{array}{l}\text { Secretaría de Turis- } \\
\text { mo, Datatur }\end{array}$ \\
\hline InATH4 & $\begin{array}{l}\text { Logaritmo natural del número de llegadas de turistas a esta- } \\
\text { blecimientos de cuatro estrellas en el destino de sol y playa } \mathrm{i} \\
\qquad \text { en el tiempo } t\end{array}$ & + & $\begin{array}{l}\text { Secretaría de Turis- } \\
\text { mo, Datatur }\end{array}$ \\
\hline InATH5 & $\begin{array}{l}\text { Logaritmo natural del número de llegadas de turistas a esta- } \\
\text { blecimientos de cinco estrellas en el destino de sol y playa i } \\
\text { en el tiempo t }\end{array}$ & + & $\begin{array}{c}\text { Secretaría de Turis- } \\
\text { mo, Datatur }\end{array}$ \\
\hline $\ln A T R 2$ & $\begin{array}{l}\text { Logaritmo natural del número de llegadas por cuarto de ho- } \\
\text { tel de dos estrellas en el destino de sol y playa i en el tiempo } \\
\text { t. Se obtiene de dividir el número de llegadas a hoteles de } \\
\text { dos estrellas entre el número de cuartos de hospedaje regis- } \\
\text { trados de establecimientos de dos estrellas }\end{array}$ & + & $\begin{array}{l}\text { Secretaría de Turis- } \\
\text { mo, Datatur }\end{array}$ \\
\hline
\end{tabular}

En tanto, la descripción de cada una de las variables, se muestra en el cuadro 3. 
- Revista de Ciencias Sociales y Humanidades. ISSN-P: 0188-9834 ISSN-E:2395-8669.

\begin{tabular}{|c|c|c|c|}
\hline Variable & Descripción & $\begin{array}{l}\text { Efecto esperado } \\
\text { sobre la tasa de } \\
\text { ocupación }\end{array}$ & Fuente \\
\hline InATR3 & $\begin{array}{l}\text { Logaritmo natural del número de llegadas por cuarto de ho- } \\
\text { tel de tres estrellas en el destino de sol y playa i en el tiempo } \\
\text { t. Se obtiene de dividir el número de llegadas a hoteles de } \\
\text { tres estrellas entre el número de cuartos de hospedaje regis- } \\
\text { trados de establecimientos de tres estrellas }\end{array}$ & + & $\begin{array}{l}\text { Secretaría de Turis- } \\
\text { mo, Datatur }\end{array}$ \\
\hline InATR4 & $\begin{array}{l}\text { Logaritmo natural del número de llegadas por cuarto de ho- } \\
\text { tel de cuatro estrellas en el destino de sol y playa i en el tiem- } \\
\text { po t. Se obtiene de dividir el número de llegadas a hoteles } \\
\text { de cuatro estrellas entre el número de cuartos de hospedaje } \\
\text { registrados de establecimientos de cuatro estrellas }\end{array}$ & + & $\begin{array}{l}\text { Secretaría de Turis- } \\
\text { mo, Datatur }\end{array}$ \\
\hline InATR5 & $\begin{array}{l}\text { Logaritmo natural del número de llegadas por cuarto de ho- } \\
\text { tel de cuatro estrellas en el destino de sol y playa i en el tiem- } \\
\text { po t. Se obtiene de dividir el número de llegadas a hoteles } \\
\text { de cinco estrellas entre el número de cuartos de hospedaje } \\
\text { registrados de establecimientos de cinco estrellas }\end{array}$ & + & $\begin{array}{l}\text { Secretaría de Turis- } \\
\text { mo, Datatur }\end{array}$ \\
\hline InST & $\begin{array}{l}\text { Logaritmo natural de la estadía promedio, se toma como } \\
\text { unidad de análisis el número de noches por persona en el } \\
\text { destino de sol y playa i en el tiempo t }\end{array}$ & + & $\begin{array}{l}\text { Secretaría de Turis- } \\
\text { mo, Datatur }\end{array}$ \\
\hline InIPC & $\begin{array}{l}\text { Logaritmo natural del Î́ndice de Precios al Consumidor por } \\
\text { región }\end{array}$ & - & INEGI \\
\hline $\ln P I B$ & Logaritmo natural del PIB por entidad federativa & + & INEGI \\
\hline$e_{\text {it }}$ & $\begin{array}{l}\text { Es el término de error y está constituido por dos componen- } \\
\text { tes. El primero es el efecto específico individual, que puede } \\
\text { ser fijo o aleatorio. El segundo es el residual e incluye la varia- } \\
\text { ción no explicada, que es espacial y temporal; es decir, aquél } \\
\text { que comúnmente se genera en econometría y es tratado } \\
\text { como estocástico. }\end{array}$ & & \\
\hline
\end{tabular}

Fuente: Elaboración propia.

Como se observa, las ecuaciones 1 y 2 son funciones de demanda, donde la cantidad requerida es la tasa de ocupación. Estas fórmulas están en función de variables de control que son sumamente relevantes: 1) El Índice Nacional de Precios al Consumidor (IPC) por región fue usado como proxy del precio; 2) El Producto Interno Bruto por entidad federativa fue usado como proxy del ingreso. Al mismo tiempo se incluyen otros factores expresados en las ecuaciones 1 y 2 , descritos en el cuadro 3.

\subsection{Técnicas econométricas empleadas}

El método de Mínimos Cuadrados Generalizados Factibles (FGLS, por sus siglas en inglés) es el estimador usado para analizar econométricamente los determinantes de la ocupación hotelera. A éste se recurre cuando la estructura de los errores no es esférica; o sea, cuando no se cumplen las hipótesis de 
homocedasticidad y ausencia de autocorrelación. Al atender estos dos inconvenientes mediante FGLS, se obtienen estimaciones insesgadas y eficientes.

Existe también la posibilidad de que se presenten inconvenientes de correlación contemporánea y de raíces unitarias. Sin embargo, pueden ser minimizados en paneles con un periodo corto.

Por ejemplo, Torres (2007) sugiere que si bien tienen impactos graves en las inferencias de macropaneles entre 20 y 30 años, no afectan en micropaneles estructurados con intervalos reducidos.

Además, no es posible verificar con precisión la existencia de estos problemas con las pruebas correspondientes y, por lo tanto, no pueden ser tratados. Lo anterior, debido a que la dimensión del tiempo es menor que las unidades transversales, o ambos son de tamaño relativamente bajo (Baltagi, 2005; Hsiao, 2003; Wooldridge, 2002).

En cuanto a la corrección de la heterocedasticidad, se presentan dos alternativas. La primera es a través de la matriz de covarianza consistente de heterocedasticidad de White (1980) para generar errores estándar robustos que, por lo general, son más grandes que los obtenidos con mínimos cuadrados ordinarios. Sin embargo, aunque se generan estimadores consistentes cuando la heterocedasticidad condicional es de forma desconocida, no son eficientes debido a que no poseen la propiedad de varianza mínima.

La segunda es mediante los estimadores FGLS y Errores Estándar Corregidos para Panel (PCSE, por sus siglas en inglés). Aunque existe una discusión importante en cuanto a la precisión de ambos, hay argumentos que favorecen al primer método, en determinadas circunstancias.

Por ejemplo, Beck y Katz (1995) refieren que es más adecuado usar FGLS cuando el número de periodos de tiempo es, por lo menos, dos veces el número de unidades transversales. De igual forma, Baltagi (2005) lo recomienda cuando el tiempo es mayor que el número de unidades transversales.

También está un trabajo más contemporáneo hecho por Reed y Ye (2011), en el que se advierten dos escenarios positivos para el uso de FGLS: 1) Cuando la intención es conseguir la eficiencia con la razón de tiempo y unidades transversales mayor o igual que 1.50 (Parks); y 2) Cuando el objetivo es lograr la eficiencia y las unidades transversales son mayores que el tiempo, ante la presencia de heterocedasticidad, medida por un coeficiente que es más alto que 1.67.

\section{Resultados y discusión}

Dentro del cuadro 4, se presenta la estadística descriptiva de las variables usadas en la evaluación econométrica de los determinantes de la tasa de ocupación. Para seleccionar la especificación econométrica correcta de los dos modelos a estimar, se realiza primero la prueba del multiplicador de Lagrange de Breusch-Pagan (LM, por sus siglas en inglés). Con ello se pretende determinar si el método adecuado es el de Efectos Aleatorios (RE, por sus siglas en inglés) o el de Regresión Agrupada (POOLED OLS, por sus siglas en inglés). La evaluación indica que existe un coeficiente con valor de cero y una probabilidad con valor de 1.

Los hallazgos obtenidos sugieren que no se puede rechazar la hipótesis nula, por lo tanto, no hay signos de efectos aleatorios. Además, los resultados no muestran evidencia suficiente para descartar la especificación de Efectos Fijos (FE, por sus siglas en inglés) como tratamiento econométrico a usar.

La estimación de la prueba F de significancia FE corrobora el rechazo de la hipótesis nula de que las 
variables dicotómicas representadas por los destinos de sol y playa son iguales a cero. Se encuentra a FE como el estimador apropiado.

A la par, se aplica la prueba de Hausman para contrastar diferencias entre RE y FE. En los dos modelos, se rechaza la hipótesis nula de que no existe correlación entre los efectos individuales y las variables explicativas, siendo preferible FE (Cuadros 4 y 5 ).

\begin{tabular}{|c|c|c|c|c|}
\hline Variable & Media & Desviación Estándar & Mínimo & Máximo \\
\hline InATH2 & 10.6474 & 1.3224 & 3.9318 & 12.9941 \\
\hline InATH3 & 11.6376 & 1.1730 & 8.1236 & 13.7473 \\
\hline InATH4 & 12.1315 & 1.4673 & 8.4888 & 14.5976 \\
\hline InATH5 & 12.5720 & 1.8185 & 7.5704 & 15.6452 \\
\hline InATR2 & 4.7665 & 0.9432 & 0.0572 & 6.3593 \\
\hline InATR3 & 4.9930 & 0.6781 & 0.0000 & 7.5018 \\
\hline InATR4 & 4.8965 & 0.9019 & 0.0000 & 10.5133 \\
\hline InATR5 & 4.6680 & 0.7722 & 0.0000 & 5.8700 \\
\hline InST & 1.0653 & 0.4287 & 0.0953 & 1.8871 \\
\hline InIPC & 4.5070 & 0.1973 & 4.1355 & 4.8138 \\
\hline $\ln P I B$ & 12.1945 & 0.7295 & 11.1604 & 13.9657 \\
\hline
\end{tabular}

Fuente: Elaboración propia.

Para verificar la presencia de heterocedasticidad entre secciones cruzadas, se efectúa la prueba del multiplicador de Wald modificada, misma que rechaza la hipótesis nula de ausencia del problema de heterocedasticidad. Para corregir el problema, los dos modelos son estimados con FGLS.

Cada uno de los coeficientes estimados de las regresiones es interpretado como elasticidades porque las variables fueron transformadas en logaritmos naturales. Los componentes llegadas de turistas por categoría de hotel y por cuarto, así como estadía de turistas en los principales destinos de sol y playa, resultan con los signos esperados, acordes a la teoría y evidencia empírica internacional (Chen et al., 2015; Ching-chi y Weng, 2010; Kim, 2010; Lei y Lam, 2015; Mutlag y Mzaiel, 2013; O’Neill, 2011; Phillips et al., 2017; Succurro y Boffa, 2010). Las variables son estadísticamente significativas a los niveles convencionales. 
A continuación, se presenta la información referente a los modelos 1 y 2 , dentro de los cuadros 5 y 6 , respectivamente.

\section{Cuadro 5. Estimaciones Modelo 1}

\begin{tabular}{|c|c|c|c|c|c|c|}
\hline \multirow[b]{2}{*}{ Variable } & \multicolumn{2}{|c|}{ RE } & \multicolumn{2}{|c|}{ FE } & \multicolumn{2}{|c|}{ FGLS } \\
\hline & $\begin{array}{l}\text { Coeficien- } \\
\text { te }\end{array}$ & Probabilidad & $\begin{array}{l}\text { Coeficien- } \\
\text { te }\end{array}$ & Probabilidad & $\begin{array}{c}\text { Coeficien- } \\
\text { te }\end{array}$ & Probabilidad \\
\hline InATH5 & $\begin{array}{c}0.0052 \\
(0.0108)\end{array}$ & 0.629 & $\begin{array}{l}0.0364 * * * \\
(0.0136)\end{array}$ & 0.009 & $\begin{array}{c}0.0238 * * * \\
(0.0084)\end{array}$ & 0.004 \\
\hline InATH4 & $\begin{array}{c}-0.0105 \\
(0.0174)\end{array}$ & 0.546 & $\begin{array}{c}0.0281 \\
(0.0190)\end{array}$ & 0.142 & $\begin{array}{l}0.0436 * * * \\
(0.0124)\end{array}$ & 0.000 \\
\hline InATH3 & $\begin{array}{c}0.0140 \\
(0.0205)\end{array}$ & 0.493 & $\begin{array}{c}0.0192 \\
(0.0229)\end{array}$ & 0.405 & $\begin{array}{l}0.0290 * * * \\
(0.0108)\end{array}$ & 0.007 \\
\hline InATH2 & $\begin{array}{l}-0.0028 \\
(0.0101)\end{array}$ & 0.780 & $\begin{array}{l}0.0180 * * \\
(0.0089)\end{array}$ & 0.047 & $\begin{array}{c}0.0212 * * * \\
(0.0046)\end{array}$ & 0.000 \\
\hline InST & $\begin{array}{l}0.1544 * * * \\
(0.0260)\end{array}$ & 0.000 & $\begin{array}{c}0.0622 \\
(0.0385)\end{array}$ & 0.110 & $\begin{array}{c}0.1114 * * * \\
(0.0228)\end{array}$ & 0.000 \\
\hline InIPC & $\begin{array}{l}0.0996^{* *} \\
(0.0490)\end{array}$ & 0.042 & $\begin{array}{c}-0.2137^{* * *} \\
(0.0814)\end{array}$ & 0.010 & $\begin{array}{c}-0.2314^{* * *} \\
(0.0457)\end{array}$ & 0.000 \\
\hline $\ln P I B$ & $\begin{array}{c}0.0082 \\
(0.0134)\end{array}$ & 0.540 & $\begin{array}{l}0.2765 * * * \\
(0.1049)\end{array}$ & 0.010 & $\begin{array}{l}0.2921 * * * \\
(0.0728)\end{array}$ & 0.000 \\
\hline Constante & $\begin{array}{l}-0.2450 \\
(0.2555)\end{array}$ & 0.338 & $\begin{array}{c}-3.1653^{* * *} \\
(1.0468)\end{array}$ & 0.003 & $\begin{array}{c}-3.2443 * * * \\
(0.6947)\end{array}$ & 0.000 \\
\hline Pruebas & & & & & & \\
\hline Breusch-Pagan & 0.00 & 1.0000 & & & & \\
\hline $\begin{array}{l}\text { Wald heterocedas- } \\
\text { ticidad }\end{array}$ & & & $1136.61^{* * *}$ & 0.0000 & & \\
\hline$F$ de efectos fijos & & & $23.87^{* * *}$ & 0.0000 & & \\
\hline Hausman & $20.67 * * *$ & 0.0043 & & & & \\
\hline Observaciones & 92 & & 92 & & 92 & \\
\hline & & & & & & \\
\hline
\end{tabular}

Fuente: Elaboración propia. Los errores estándar se presentan en paréntesis. *Significancia al 10\%; **Significancia al 5\%; ***Significancia al $1 \%$. 
Cuadro 6. Estimaciones Modelo 2

\begin{tabular}{|c|c|c|c|c|c|c|}
\hline \multirow{2}{*}{ Variable } & \multicolumn{2}{|c|}{ RE } & \multicolumn{2}{|c|}{ FE } & \multicolumn{2}{|c|}{ FGLS } \\
\hline & Coeficiente & Probabilidad & Coeficiente & Probabilidad & Coeficiente & Probabilidad \\
\hline InATR5 & $\begin{array}{c}0.0012 \\
(0.0147)\end{array}$ & 0.935 & $\begin{array}{l}0.0274^{* *} \\
(0.0120)\end{array}$ & 0.025 & $\begin{array}{c}0.0132 * \\
(0.0078)\end{array}$ & 0.089 \\
\hline InATR4 & $\begin{array}{c}0.0163 \\
(0.0186)\end{array}$ & 0.379 & $\begin{array}{c}0.0175 \\
(0.0136)\end{array}$ & 0.200 & $\begin{array}{l}0.0262 * * * \\
(0.0073)\end{array}$ & 0.000 \\
\hline InATR3 & $\begin{array}{c}-0.0047 \\
(0.0246)\end{array}$ & 0.848 & $\begin{array}{l}0.0641 * * * \\
(0.0226)\end{array}$ & 0.006 & $\begin{array}{c}0.0623^{* * *} \\
(0.0139)\end{array}$ & 0.000 \\
\hline InATR2 & $\begin{array}{c}0.0074 \\
(0.0094)\end{array}$ & 0.431 & $\begin{array}{l}0.0176^{*} \\
(0.0091)\end{array}$ & 0.057 & $\begin{array}{l}0.0244^{* * *} \\
(0.0042)\end{array}$ & 0.000 \\
\hline InST & $\begin{array}{c}0.1797 * * * \\
(0.0208)\end{array}$ & 0.000 & $\begin{array}{l}0.0623 * \\
(0.0378)\end{array}$ & 0.104 & $\begin{array}{l}0.1063^{* * *} \\
(0.0234)\end{array}$ & 0.000 \\
\hline $\operatorname{lnIPC}$ & $\begin{array}{l}0.1171^{* *} \\
(0.0466)\end{array}$ & 0.012 & $\begin{array}{c}-0.2289 * * * \\
(0.0780)\end{array}$ & 0.004 & $\begin{array}{c}-0.2146 * * * \\
(0.0445)\end{array}$ & 0.000 \\
\hline $\ln P I B$ & $\begin{array}{c}0.0015 \\
(0.0124)\end{array}$ & 0.907 & $\begin{array}{l}0.3527 * * * \\
(0.0979)\end{array}$ & 0.001 & $\begin{array}{l}0.3270 * * * \\
(0.0653)\end{array}$ & 0.000 \\
\hline Constante & $\begin{array}{l}-0.2929 \\
(0.2373)\end{array}$ & 0.217 & $\begin{array}{c}-3.4264 * * * \\
(0.9440)\end{array}$ & 0.001 & $\begin{array}{c}-2.9265^{* * *} \\
(0.6115)\end{array}$ & 0.000 \\
\hline Pruebas & & & & & & \\
\hline Breusch-Pagan & 0.00 & 1.0000 & & & & \\
\hline $\begin{array}{l}\text { Wald heterocedas- } \\
\text { ticidad }\end{array}$ & & & $768.86 * * *$ & 0.0000 & & \\
\hline$F$ de efectos fijos & & & $15.57 * * *$ & 0.0000 & & \\
\hline Hausman & $89.99 * * *$ & 0.0000 & & & & \\
\hline Observaciones & 91 & & 91 & & 91 & \\
\hline
\end{tabular}

Fuente: Elaboración propia. Los errores estándar se presentan en paréntesis.

*Significancia al 10\%; $* *$ Significancia al 5\%; $* * *$ Significancia al 1\%. 
Referente a las llegadas de turistas a las distintas categorías de hoteles, se comprueba la hipótesis de que los lugares de sol y playa que reciben más visitantes en los establecimientos impactan más en la tasa de ocupación. Lo anterior es acorde con la evidencia empírica internacional (Ching-chi y Weng, 2010; Kim, 2010; Lei y Lam, 2015; Succurro y Boffa, 2010).

En cuanto a hoteles de cuatro estrellas, éstos registran la mayor magnitud. Además, los resultados sugieren que un incremento de $1 \%$ en la llegada de turistas, aumenta la tasa de ocupación en $0.04 \%$. La mayor aportación a esta tasa deriva de las llegadas de visitantes en el estrato de cuatro estrellas, que es el sector más dinámico y el que experimenta mayores tasas de crecimiento con respecto al resto.

Las diferencias en las tasas de ocupación entre cada una de las clasificaciones están asociadas a que los agentes económicos prefieren categorías de hotel relativamente más accesibles en precio y que se ajusten a las necesidades de ubicación, excelencia en el servicio, productos ofrecidos, infraestructura, entre otras (Jeffrey y Barden, 2001; Jones y Chen, 2011; Mei Fung et al., 2016; Phillips et al., 2017).

Aunque existen diferencias en cuanto a calidad del producto y estándares de servicio en cada categoría de hotel, es evidente que los turistas eligen el tipo de establecimiento que es más acorde con su restricción presupuestaria (Jeffrey et al., 2002).

Pese a que los lugares de sol y playa abordados en el estudio cuentan principalmente con hoteles cinco estrellas, no significa que deba promocionarse en mayor medida este estrato. Más aún, debe incentivarse la creación de más infraestructura en establecimientos de cuatro estrellas, ya que no cuentan con la misma capacidad de los primeros para absorber el hospedaje.

Paralelamente, los hoteles cinco estrellas deben ofrecer estímulos a los consumidores para que se inclinen por este rubro, de tal manera que se logre descargar la demanda de los establecimientos de cuatro estrellas.

Respecto a las llegadas de turistas por cuarto, se registra también un efecto positivo sobre la tasa de ocupación hotelera. Este resultado es acorde con los trabajos empíricos internacionales de Ching-chi y Weng (2010), Kim (2010), Lei y Lam (2015), Mutlag y Mzaiel (2013), quienes usaron como variable cuartos disponibles totales en sus modelos de regresión.

Aquí, el estrato de tres estrellas alcanza el mayor coeficiente con $0.06 \%$ y es estadísticamente significativo al $1 \%$. En segundo lugar, se ubica la categoría de cuatro estrellas, con una diferencia de coeficiente de casi $0.04 \%$, con respecto al primer estrato mencionado. Es evidente que el primer lugar de preferencia, en ambos casos, dependerá del refinamiento del modelo, que consiste en controlar la cifra de llegadas por número de cuartos.

La estadía también muestra consistencia en los dos modelos con coeficientes de $0.11 \%$. De acuerdo con la evidencia empírica, este factor actúa como estabilizador de la tasa de ocupación y se convierte en un incentivo determinante para los desarrolladores de hoteles (Jeffrey y Barden, 2001; O’Neill, 2011).

En una investigación realizada para la ciudad de Madrid (Esteban y Reinares, 2008), se observa que dos de los atributos más valorados por los turistas son la calidad y el precio. Los dos contribuyen a prolongar el alojamiento en determinado establecimiento. En este punto, destacan sobre todo los hoteles con categorías de tres y cuatro estrellas.

En el caso de las variables de control como el IPC y el PIB, ambas presentan los signos acordes a la teoría económica. El IPC, que es proxy del precio, arroja un coeficiente negativo debido a que existe una relación inversa entre el precio y la cantidad demandada. En tanto, el PIB, que es proxy del ingreso, presenta el coeficiente con signo positivo, ya que existe una relación directa entre el ingreso y la cantidad demandada. 
Cabe mencionar que dos limitantes del presente trabajo se deben, primeramente, a que no es posible discernir el impacto de la estadía por categoría de hotel, pues no se encuentra información desagregada a ese nivel; asimismo, a que no se cuenta con datos sobre los efectos estacionales, debido a que las series están anualizadas

Subsanar ambos aspectos, respectivamente, sería de gran valía en la construcción de políticas públicas y en la planeación y operación de estrategias dentro de la industria hotelera para mejorar el desempeño de la tasa de ocupación; así como para la toma de decisiones sobre el establecimiento de tarifas diferenciadas y el ofrecimiento de paquetes en temporada baja y alta.

\section{Conclusión}

De acuerdo con los datos de la investigación, se cumple con el objetivo de estudiar la relación entre variables turísticas clave con la tasa de ocupación hotelera. Del mismo modo, se confirma la hipótesis de que los centros turísticos donde se hospedan más visitantes por categoría de hotel y por tipo de cuarto, y amplían su alojamiento, tienden a contribuir con más intensidad en la tasa de ocupación.

Las variables de control como el IPC y el PIB revelan signos que son acordes con la teoría económica. El primer coeficiente es negativo debido a la relación inversa que existe entre cantidad demandada y precio. En el caso del segundo, se observa un signo positivo, ya que existe una relación directa entre el ingreso y la cantidad demandada. El hecho de que los resultados de los coeficientes de la estadía, el IPC y el PIB son similares, significa que los datos obtenidos son sólidos.

Con el análisis hecho en este trabajo, también se puede comprender la relación que guardan las variables turísticas básicas asociadas al desempeño turístico reciente de los centros de playa.

Es pertinente señalar que el hospedaje en hoteles de tres y cuatro estrellas refleja mayor magnitud en la tasa de ocupación que el resto. Como una mejora al servicio, se recomienda que el desarrollo del turismo se enfoque en el crecimiento de infraestructura de establecimientos que cuenten con estas categorías, debido a que son los segmentos con menos capacidad para cubrir la creciente demanda de alojamiento de visitantes.

Conjuntamente, es necesario no descuidar la política de generación de incentivos para que los usuarios también se decidan por hoteles de cinco estrellas. Esto es importante, pues este tipo de infraestructura es lo que caracteriza a un destino turístico de hegemonía.

Otro punto relevante es que los datos recabados a lo largo de este trabajo se constituyen como información cuantitativa muy significativa. Éstos pueden ser tomados como referencia en la elaboración de propuestas que contribuyan a un crecimiento más equilibrado de la ocupación hotelera y en la generación de indicadores turísticos básicos de los destinos de sol y playa de México, a fin de aminorar las asimetrías detectadas.

No obstante que este trabajo se limita al estudio concreto de los determinantes de la ocupación hotelera en sitios de playa y toma como referencia su relación con indicadores específicos; resulta evidente la existencia de factores psicosociales, ambientales, económicos, culturales y legales que influyen en el comportamiento de la variable aquí analizada.

Para futuros análisis se recomienda llevar a cabo comparativos por tipo de destino; de igual forma, se incorporen más parámetros cualitativos y cuantitativos (por ejemplo, la ocupación por categoría según 
el origen del visitante). Al hacerlo se podrán comprender con mayor precisión y amplitud las causas del comportamiento de la variable y de los sitios estudiados; además de dotar a los tomadores de decisiones con información que les ayude a implementar estrategias con mayores posibilidades de éxito.

Por último, en investigaciones posteriores se debe considerar el impacto que tiene la entrada en vigor de los Lineamientos del Sistema de Clasificación Hotelera, en el reordenamiento del número de los establecimientos de hospedaje por categoría, dentro de los niveles de ocupación, estadía, afluencia de visitantes y otros; debido a que son variables clave para el crecimiento de la industria de hospedaje en México.

\section{Referencias}

Amaya, Carlos, Ana Sosa y Pedro Moncada. 2017. Determinantes de competitividad turística en destinos de sol y playa mexicanos. Región y sociedad, 29 (68): 279-315.

Arbelo, Antonio, Marta Arbelo-Pérez y Pilar Pérez-Gómez. 2018. Estimation of profit efficiency in the hotel industry using a Bayesian stochastic frontier model. Cornell Hospitality Quarterly, 59 (4): 1-12. DOI: $10.1177 / 1938965518762841$.

Baltagi, Badi. 2005. Econometric Analysis of Panel Data. Londres: John Wiley \& Sons.

Beck, Nathaniel y Jonathan Katz. 1995. What to do (and not to do) with time-series cross-section data. American Political Science Review, 89 (3): 634-647.

Benseny, Graciela. 2007. El turismo en México. Apreciaciones sobre el turismo en espacio litoral. Aportes $y$ Transferencias, 11 (2): 13-34.

Bringas, Nora. 1999. Políticas de desarrollo turístico en dos zonas del pacífico mexicano. Región y sociedad, 11 (17): 3-52.

Chen, Chiang-Ming, Ho-Wen Yang, Eldon Li y Chia-Chang Liu. 2015. How does hotel pricing influence guest satisfaction by the moderating influence of room occupancy. International Journal of Hospitality Management, 49:136-138. DOI: http://dx.doi.org/10.1016/j.ijhm.2015.06.006

Ching-chi, Cindia y Clara Weng. 2010. Hotel Occupancy Rate in a Gambling Destination: A Longitudinal Study of the Macao Hotel Industry 2004-2007, Journal of Hospitality and Tourism Management, 17: 34-43. DOI: 10.1375/jhtm.17.1.34.

Coll-Hurtado, Atlántida. 2016. Espacio y ocio: el turismo en México. Ciudad de México: Universidad Nacional Autónoma de México.

CONAPO. 2016. Ingresos por divisas a México según fuente, 1990-2016. http://www.conapo.gob.mx/ es/OMI/Ingresos_por_divisas_a_Mexico_segun_fuente_1990_2016 (2 de mayo, 2019).

Cruz-Chávez, Gustavo, Víctor Hernández-Trejo, Gerzaín Avilés-Polanco y Ramón Valdivia-Alcalá. 2016. Turismo extranjero y crecimiento económico en México. Evidencia empírica para Los Cabos. Economía, Sociedad y Territorio, 16 (50): 1-38.

Damián, Ionela y Enrique Navarro. 2005. Estudio de la capacidad de carga social percibida por los turistas aplicado en la costa del sol oriental. Reporte del Proyecto SEJ-2005-04660: España: Universidad de Málaga, Almería, Alicante, Pablo Olavide (Sevilla) y Novo Lisboa (Portugal).

Datatur. 2019. Reporte monitoreo hotelero 70 centros turísticos marzo 2019. https://www.datatur.sectur. gob.mx/SitePages/ActividadHotelera.aspx (4 de mayo de 2019). 
Davila, Arturo. 2015. Centros Integralmente Planeados (CIPS) en México. El proyecto turístico del FONATUR. Cuadernos de investigación en urbanismo, 5-6 (17): 270-285.

Esteban, Agueda y Eva Reinares. 2008. Análisis del comportamiento de la demanda hotelera y su posible interferencia por el sistema de clasificación de la oferta. Madrid: Universidad Complutense de Madrid.

Ging, Chew. 2010. The dynamic interactions between hotel room rates and international inbound tourists: evidence from Singapore. International Journal of Hospitality Management, 29: 758-760. DOI:10.1016/j.ijhm.2009.09.005

2011. The determinants of hotel room rates: another visit with Singapore's data. International Journal of Hospitality Management, 30: 756-758. DOI:10.1016/j.ijhm.2010.09.010.

Gómez, Salvador. 2011. La planificación de los destinos turísticos mexicanos: una receta mil veces vanagloriada. Revista Investigaciones Turísticas, 4: 1-21.

Guzmán-Soria, Eugenio, María de la Garza, Samuel Rebollar-Rebollar, Juvencio Hernández-Martínez y José García-Salazar. 2011. Factores determinantes de la demanda internacional del turismo en México. Revista de Globalización, Competitividad y Gobernabilidad, 5 (3): 30-49.

Hsiao, Cheng. 2003. Analysis of Panel Data. Cambridge, United Kingdom: Cambridge University Press. Ibáñez, Reyna. 2016. Divergencias asociadas al desarrollo turístico en destinos costeros de la región noroeste de México. Revista Iberoamericana de Turismo- RITUR, 6 (2): 63-91.

2017. Propuesta de indicadores alternos, a partir de un análisis basado en indicadores convencionales y las asimetrías en municipios turísticos con destinos de sol y playa. Nova Scientia, 9 (19): 850-901.

Jang, SooCheong y Larry Yu. 2002. Analysis of return on hotel investment: a comparison of commercial hotel companies and casino hotel companies. Journal of Hospitality and Tourism Research, 26 (1): 38-53. Jeffrey, Douglas y Robin Barden. 2000. Monitoring hotel performance using occupancy time-series analysis: the concept of occupancy performance space. International Journal of Tourism Research, 2: 383-402. ------ 2001. Multivariate models of hotel occupancy performance and their implications for hotel marketing. International Journal of Tourism Research, 3: 33-44.

Jeffrey, Douglas, Robin Barden, Peter Buckley y Nicolas Hubbard. 2002. What Makes for a Successful Hotel? Insights on Hotel Management Following 15 Years of Hotel Occupancy Analysis in England. The Service Industries Journal, 22 (2): 73-88.

Jiménez, Alfonso. 1988. Desarrollo turístico y sustentabilidad: el caso de México. Ciudad de México: Porrúa.

Jones, Peter y Meng-Mei Chen. 2011. Factors determining hotel selection: Online behaviour by leisure travelers. Tourism and Hospitality Research, 11 (1): 83-95. Doi: 10.1057/thr.2010.20

Kim, Hong. 2010. Hotel property characteristics and occupancy rate: Examining super Deluxe 1st class hotels in Seoul, Korea. International Journal of Tourism Sciencies, 10 (3): 25-47.

Koenig, Nicole y Eberhard Bischoff. 2004. Analyzing seasonality in welsh room occupancy data. Annals of Tourism Research, 31 (2): 374-392. DOI:10.1016/j.annals.2003.12.006

Lam, Cindia y Clara Lei. 2010. Hotel occupancy rate in a gambling destination: a longitudinal study of the Macao hotel industry 2004-2007. Journal of Hospitality and Tourism Management, 17: 34-43. DOI: 10.1375/jhtm.17.1.34 
Lei, Clara y Cindia Lam. 2015. Determinants of hotel occupancy rate in a Chinese gaming destination, Journal of Hospitality and Tourism Management, 22: 1-9. DOI: http://dx.doi.org/10.1016/j. jhtm.2014.12.003

Lime, David. 1977. Principles of Recreation Carrying Capacity. En Proceedings of the Southern States Recreation Applications, compilado por D. Lime. North Carolina: Asheville, 122-134.

Lucas, Robert. 1964. The recreational capacity of the Quetico-Superior Area. Washington, D.C.: Departament of Agriculture, National Agricultural.

Madrid, Francisco. 2015. La sostenibilidad en la política turística mexicana. PASOS. Revista de Turismo y Patrimonio Cultural, 13 (6): 1301-1313.

Mathew, Sean. 2016. A study of large hotel occupancy rates on the island of St. Lucia. Tesis de Doctorado, Minneapolis, Minnesota: Walden University.

Mei Fung, Candy, Nada Kulendran, Brian King y Mathew Yap. 2016. Research note: using demand determinants to anticipate fluctuations in hotel occupancy. Tourism Economics, 22 (1): 179-187.

Mutlag, Abdul y Noorya Mzaiel. 2013. Factors affecting hotels occupancy rate (an empirical study on some hotels in Amman. Interdisciplinary Journal of Contemporary Research in Business, 5 (6): 142-159.

O'Neill, John. 2011. Hotel occupancy: is the three-year stabilization assumption justified. Cornell Hospitality Quarterly, 52 (2): 176-180. DOI: 10.1177/1938965510393733.

Peng, Kua-Hsin, Jen-Hung Huang y Wen-Hsiung Wu. 2013. Rasch model in data envelopment analysis: application in the international tourist hotel industry. Journal of the Operational Research Society, 64: 938-944. DOI: https://doi.org/10.1057/jors.2012.97.

Phillips, Paul, Stuart Barnes, Krystin Zigan y Roland Schegg. 2017. Understanding the Impact of Online Reviews on Hotel Performance: An Empirical Analysis. Journal of Travel Research, 56 (2): 235-249. DOI: $10.1177 / 0047287516636481$.

Qu, Hailin, Peng Xu y Amy Tan. 2002. A simultaneous equations model of the hotel room supply and demand in Hong Kong. International Journal Hospitality Management, 21: 455-462.

Reed, Robert y Haichun Ye. 2011. Which panel data estimator should I use?. Applied Economics, 43 (8): 985-1000. DOI: http://dx.doi.org/10.1080/00036840802600087.

SECTUR. 2014. Centros de Playa. http://www.sectur.gob.mx/programas/programas-regionales/centrosde-playa/ (7 de mayo, 2019).

Succurro, Marianna y Federico Boffa. 2010. Search cost reduction increases variation in hotels occupancy rate: A theoretical and empirical analysis. Arcavacata, Italia: Università Della Calabria.

Torres, Oscar. 2007. Panel Data Analysis, Fixed \& Random Effects. Princeton: Princeton University. Tulio, Marco y Arturo Santamaría. 2015. Los centros integralmente planeados (CIP’s) en México. Revista Latino-Americana de Turismologia, 1 (1): 36-53.

Velarde, Mónica, María Santillán y Kennedy Obombo. 2016. Estrategias determinantes para la competitividad de un destino de sol y playa. El caso de Mazatlán, Sinaloa, México. Investigaciones Turísticas, 11: 116-142.

White, Halbert. 1980. A Heteroskedasticity-Consistent Covariance Matrix Estimator and a Direct Test for Heteroskedasticity. Econometrica, 48 (4): 817-838.

Wooldridge, Jeffrey. 2002. Econometric Analysis of Cross Section and Panel Data. Cambridge, Massachusetts: The MIT Press. 